\title{
Nucleotide-sugar transporters: structure, function and roles in vivo
}

\section{Handford ${ }^{1}$, \\ C. Rodriguez-Furlán ${ }^{2,3}$ and A. Orellana ${ }^{2,3}$}

\author{
${ }^{1}$ Department of Biology, Faculty of Science, University of Chile, Santiago, Chile \\ ${ }^{2}$ Millenium Nucleus in Plant Cell Biology, ${ }^{3}$ Center of Plant Biotechnology, \\ Andres Bello University, Santiago, Chile
}

\section{Correspondence \\ A. Orellana \\ Center of Plant Biotechnology \\ Andres Bello University \\ República, 217 \\ Santiago \\ Chile \\ Fax: +56-2-661-5832 \\ E-mail: aorellana@unab.cl \\ Presented at the XVIII Annual Meeting of the Federação de \\ Sociedades de Biologia \\ Experimental, Curitiba, PR, \\ Brazil, August 27-30, 2003. \\ Research supported by The Leverhulme Trust (M. Handford), Fondecyt (No. 19030551) and Iniciativa Científica Milenio P02-009-F.}

Received October 5, 2005

Accepted June 6, 2006

Accepted june 6, $2006, \ldots \ldots$

\begin{abstract}
The glycosylation of glycoconjugates and the biosynthesis of polysaccharides depend on nucleotide-sugars which are the substrates for glycosyltransferases. A large proportion of these enzymes are located within the lumen of the Golgi apparatus as well as the endoplasmic reticulum, while many of the nucleotide-sugars are synthesized in the cytosol. Thus, nucleotide-sugars are translocated from the cytosol to the lumen of the Golgi apparatus and endoplasmic reticulum by multiple spanning domain proteins known as nucleotide-sugar transporters (NSTs). These proteins were first identified biochemically and some of them were cloned by complementation of mutants. Genome and expressed sequence tag sequencing allowed the identification of a number of sequences that may encode for NSTs in different organisms. The functional characterization of some of these genes has shown that some of them can be highly specific in their substrate specificity while others can utilize up to three different nucleotidesugars containing the same nucleotide. Mutations in genes encoding for NSTs can lead to changes in development in Drosophila melanogaster or Caenorhabditis elegans, as well as alterations in the infectivity of Leishmania donovani. In humans, the mutation of a GDP-fucose transporter is responsible for an impaired immune response as well as retarded growth. These results suggest that, even though there appear to be a fair number of genes encoding for NSTs, they are not functionally redundant and seem to play specific roles in glycosylation.
\end{abstract}

Key words

- Glycoconjugates

- Glycosyltransferases

- Golgi apparatus

- Nucleotide-sugars

- Nucleotide-sugar

transporters

\section{Introduction}

Cell surface glycoconjugates - polysaccharides, glycoproteins, and glycolipids play many fundamental roles in the growth and development of uni- and multicellular eukaryotes, including yeast, plants, rats, and humans (1-3). In addition, cell surface recognition of hosts by their pathogens such as Candida spp and Leishmania spp also re- quires these molecules (4). The glycan portion of glycoproteins, proteoglycans and polysaccharides is synthesized and modified by glycosyltransferases located in the lumen of the endoplasmic reticulum (ER) and Golgi apparatus. The substrates required by all glycosyltransferases are sugars activated by the addition of a nucleoside mono- or diphosphate (UDP, GDP, or CMP) forming a nucleotide-sugar. 
In most organisms, the vast majority of nucleotide-sugars are synthesized in wellcharacterized reactions from NDP-glucose (glc) (5). These reactions take place predominantly in the cytosol. Nevertheless, considering that the substrates required by the glycosyltransferases are synthesized in the cytosol, although the active site of the glycosyltransferases is located in the Golgi/ER lumen, and given the highly unfavorable task for a molecule to directly cross the lipid bilayer, the existence of nucleotide-sugar transporter (NST) proteins has been proposed (6).

\section{Biochemical evidence for the existence of nucleotide-sugar transporters}

Initial experiments leading to the discovery of NSTs began thirty years ago (7-9). When Golgi vesicles isolated from rat mammary glands were incubated with UDP-galactose (gal) and UDP-glc, lactose was synthesized in the lumen. A cycle was proposed whereby UDP sugars enter the Golgi compartment and the sugar is transferred by the glycosyltransferase to the nascent glycan (in this case lactose). Subsequently, UDP is hydrolyzed. The inorganic phosphate leaves the lumen by an unknown mechanism, whereas the UMP exits the compartment in antiport with the incoming nucleotide-sugar

Figure 1. Model for the topology of glycosylation reactions in the Golgi/endoplasmic reticulum (ER) apparatus. Nucleotide-sugars are imported into the lumen from the cytosol via specific transporter proteins. Glycosyltransferase enzymes polymerize the addition of the sugar portion to glycoconjugates. The resulting NDP is cleaved by a luminal NDPase forming inorganic phosphate $(\mathrm{Pi})$ and NMP, the latter of which exits in antiport with the incoming nucleotide-sugar. The precise exit mechanism of inorganic phosphate is unknown.
(Figure 1).

Further evidence for this model was subsequently obtained (6). The concentration of UDP- $N$-acetyl glucosamine (glcNAc) in the lumen of vesicles isolated from rat liver was 20 times the concentration in the bathing medium. Furthermore, UDP-glcNAc import could be stimulated by mechanically preloading the vesicles with UMP (10). This uptake required the presence of intact vesicles since transport of the nucleotide-sugar fell substantially when vesicles were permeabilized with a detergent. These experiments and those of others implied that nucleotidesugar uptake was protein mediated and should therefore meet several requirements (11). Uptake should be temperature dependent and saturable; typically the $K_{\mathrm{m}}$ for nucleotide-sugar import is in the range of $1-10 \mu \mathrm{M}$. Moreover, uptake should be sensitive to proteases - if vesicles are pretreated with a protease prior to incubation with the nucleotide-sugar, incorporation into glycans falls. However, in plants the reduction can be overcome if the vesicles are subsequently permeabilized with a nonionic detergent such as Triton X100, with the substrate thus having direct access to the glycosyltransferases for the synthesis of polysaccharides (for example, see Ref. 12). In addition, the biochemical analyses with proteases hinted at the structure of the NSTs in that at least part of the protein must be exposed to the cytosol for successful transport of the substrate and this region is essential for transport.

In general, the range of nucleotide-sugars transported into the lumen reflects the diversity of sugar structures present on the glycoconjugates, i.e., sugars not found in the lumen are not transported. For example, UDP-glc is transported into rat ER vesicles, whereas non-metabolized GDP-mannose (man) is not (13). Several biochemical experiments have demonstrated that multiple NSTs exist in each organism, and that there is not simply a single protein accounting for the transfer across the membrane of all 
nucleotide-sugars required by glycosyltransferases. For example, in plants, UDP-glc and GDP-fucose (fuc), both of which are required for glycosylation reactions in peas (Pisum sativum), are transported independently into the Golgi lumen (14).

\section{Molecular and structural analysis of nucleotide-sugar transporters}

These biochemical studies have been greatly complemented by the subsequent molecular cloning and characterization of NSTs, which have now been identified in a wide range of model species, such as Saccharomyces cerevisiae, Kluyveromyces lactis, Candida spp, the protozoan parasite Leishmania donovani, Arabidopsis thaliana, Drosophila melanogaster, rats, and humans. The first were identified and characterized a decade ago by complementing mutants that were known to be defective in nucleotidesugar transport in the Golgi apparatus (1517). In addition, other NST genes such as $S c V R G 4, S Q V-7, F R C$, and $L P G 2$ were identified by mapping genes that were responsible for specific glycosylation defects (for reviews, see Refs. 18 and 19). By using these NST gene sequences a number of putative NST genes were identified in the genome of different species. Some of them have been functionally characterized but the function of a large number of these genes remains unknown. Among the features shared by the predicted NST proteins are the length (300350 amino acids) and thus molecular weight. In addition, these proteins are highly hydrophobic and the use of algorithms to estimate the number of transmembrane domains predicts the presence of 6-10 transmembrane $\alpha$ helical spans, consistent with their role in transferring substrates across the membrane $(4,20,21)$. This feature was confirmed experimentally for the mouse CMP-sialic acid (CMP-sia) transporter (22) that contains 10 transmembrane domains. Furthermore, most predictions determine an even number of spans, so that the $\mathrm{N}$ - and $\mathrm{C}$-termini would be on the same side of the membrane. Such in silico analyses have been supported by experimental evidence demonstrating that both the $\mathrm{N}$ - and C-termini are in the cytosolic compartment for the CMP-sia and GDPman $(\mathrm{ScVrg} 4 \mathrm{p})$ transporters from mice and yeast, respectively $(22,23)$. Interestingly, the presence of the $\mathrm{N}$-terminal cytosolic domain seems to be essential for the correct targeting of NSTs since the yeast GDP-man transporter, $\mathrm{ScVrg} 4 \mathrm{p}$, is retained in the ER when this domain is removed (23).

\section{Substrate specificity of nucleotide-sugar transporters}

Many studies have been undertaken to characterize the specificity of NSTs. All of these investigations were limited to the study of those nucleotide-sugars which are available in radio-labeled form. To date, those available commercially do not yet reflect the broad range of substrates found in vivo in different organisms. Despite this limitation, progress in the field has been significant and some trends are appearing. Initially, it was believed that NSTs were monospecific, transporting a single nucleotide-sugar across the membrane. For example, the mouse CMPsia transporter is only able to transport this nucleotide-sugar even though this NST shows greater sequence similarity to UDPgal transporters (24). Additionally, in Arabidopsis, AtUTr2 is only capable of transporting UDP-gal (25). However, such monospecificity is becoming the exception, with more and more NSTs with the ability to transport multiple substrates being identified. Functional complementation of organisms with defective nucleotide-sugar transport and the reconstitution of NSTs into proteoliposomes have proved to be very effective tools for the analysis of the substrate specificity of the antiporters. $L P G 2$ from Leishmania was the first multi-specific transporter identified, capable of transporting 
GDP-man, GDP-fuc and GDP-arabinose $(26,27)$. NSTs capable of transporting multiple UDP-sugars have been subsequently found in multicellular eukaryotes, including SQV-7 in C. elegans (28), FRC of Drosophila (29,30), a human UDP-glucuronic acid/ UDP- $N$-acetyl galactosamine transporter (glcA/galNAc) (31), and AtUTr1 of Arabidopsis (21). In all cases of multi-specificity, the sugars transported are limited to those attached to either UDP or GDP, and NSTs able to transport both UDP- and GDP-sugars have not been identified. Lending further support to the notion that the nucleotide portion plays the most significant role in binding to the NST is the observation that nucleotide-sugar import can be inhibited by raising the free NDP concentration, but is unaffected if the free-sugar concentration is elevated (32). The creation of chimeric proteins between the human CMP-sia and UDPgal transporters has shown that artificial NSTs can be generated which are capable of transporting both nucleotide sugars $(33,34)$.

\section{Structure-function relationship}

Berninsone and Hirschberg (35) showed that sequence similarity is not sufficient to predict the function of an NST. Therefore, in order to identify protein domains that would permit us to determine a relationship between function and protein sequence, we performed a phylogenetic analysis of NST
Figure 2. Phylogenetic tree of nucleotide-sugar transporters. The tree was constructed using Clustal $X$ and the Mega software. Method: Neighbor joining; Model: p-distance; Bootstrap: replications 100 .

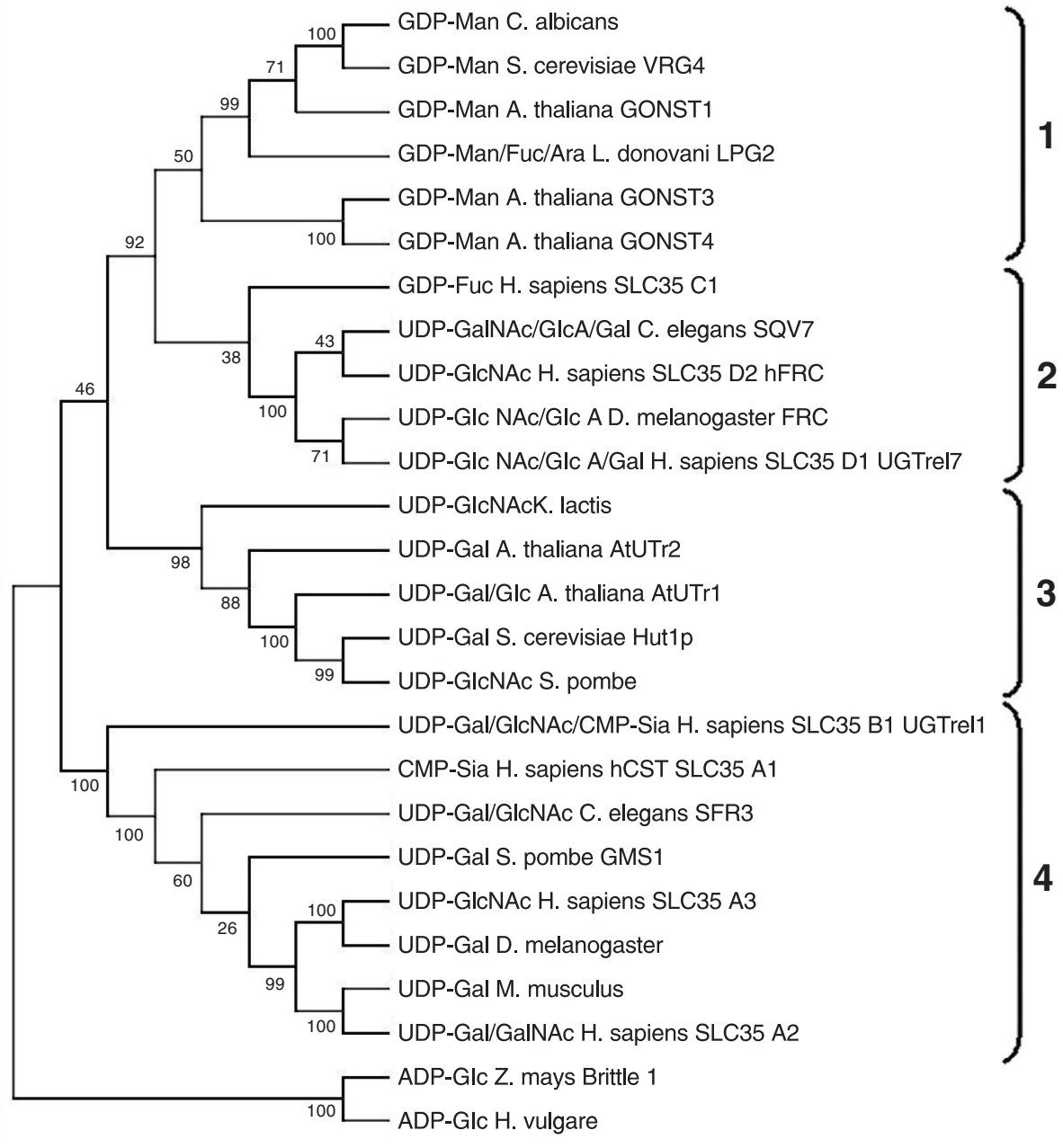


genes that had been already characterized and analyzed each clade (Figure 2). The results indicated that they can be separated into different groups with some functional similarities. The first clade is formed by GDP-sugar transporters from different organisms such as yeast, Candida and Arabidopsis. A conserved region is found among the NSTs present in this group (Figure 3). Yeast studies have shown that mutations in the GX (L/V)NK motif dramatically reduce the ability of ScVrg4p to transport GDPman. Moreover, photoaffinity-binding studies have shown that this motif binds to the GDP moiety of the substrate (36) and the presence of this motif helped to identify putative NSTs capable of transporting GDPman in Candida albicans, C. glabrata and Arabidopsis (20,37-39).

The second group within the tree is formed by NSTs that transport UDP-sugars, some of which have been classified as multisubstrate NSTs ( $S Q V-7$ and $F R C$ ). However, although more distantly related, this group also contains a GDP-fucose transporter, suggesting a weak relationship between primary structure and substrate specificity. Interestingly, lysine 303 and threonine 308 located near the C-terminus of the GDP-fucose transporter have been proposed to play a role in the recognition of the substrate. However, even though these two residues are also present in other NSTs belonging to this group, they are located in a region that shows a high probability to be part of a predicted transmembrane domain (Figure 4). Therefore, the exposure of this domain may be important for substrate recognition.

The third group consists of NSTs that transport one or two substrates from a restricted number of nucleotide sugars (UDPglucose, UDP-galactose and UDP-glcNAc). Some of these NSTs have di-lysine motifs that are involved in the retention of membrane proteins in the ER. Recent data indicate that AtUTr1 is in fact located in the ER

\section{C. albicans VRG4 GONST3 \\ GONST 4 \\ GONST1 \\ LPG2}

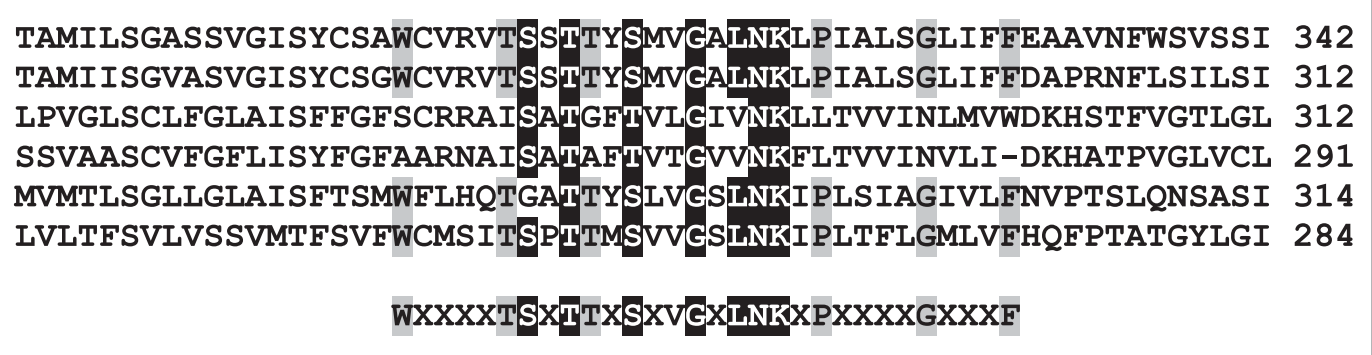

Figure 3. Conserved sequence found in GDP-sugar transporters. Alignment using the Clustal W program. The pattern contains the GXLNK sequence that binds GDP. The highly conserved sequences are boxed in black while the less conserved ones are boxed in gray.
SQV-7 C. elegans FRC D. melanogaster UDP-GlcA/GalNAC [Hs] GDP-Fucose [Hs] UDP-GICNAC [Hs ]

\section{TMD}

TMD

VWTCFLLSCICGFVLNYSLVLCTHHNSALTTTCVGPIKNLFVTYVGMFSS FVVQFLLSCVMGFILSYSTILCTQFNSALTTTIVGCLKNICVTYLGMFIG FLLQFTLSCVMGF ILMYATVLCTQYNSALTTTIVGCIKNILITYIGMVFG FWGMMTLGGLFGFAIGYVTGLQIKFTSPLTHNVSGTAKACAQTVLAVLYY TWIVVVLQALGGLVIAAVIKYADNILKGFATSLS----IILSTLISYFWL

Figure 4. Conserved residues in nucleotide-sugar transporters from group 2. The conserved residues $\mathrm{K}$ and T present in the NSTs that belong to group 2 are boxed in black. It can be observed that these residues are part of putative transmembrane domains (TMD) except for the human GDP-fucose transporter. 
(40). In addition, the expression of this NST is up-regulated under conditions that stimulate the unfolded protein response (40), a mechanism that is activated when an accumulation of unfolded proteins occurs in the ER (41). The folding of glycoproteins in this organelle depends on calnexin and calreticulin, two chaperones that retain unfolded monoglucosylated glycoproteins formed by the UDP-glucose glycoprotein glycosyltransferase (UGGT), an enzyme that reglucosylates unfolded proteins using UDP-glucose as substrate (42). This reglucosylation process occurs until the glycoprotein is completely folded and therefore, AtUTr1 is likely to be involved in supplying the UDP-glucose required by UGGT during the folding of glycoproteins in the ER. Hence, AtUTr1 may not be involved in the biosynthesis of glycoconjugates but may be involved in a regulatory process that is a key step in the biosynthesis of glycoproteins. Some of the NSTs that belong to this group (AtUTr1, ScHut1p) contain in their C-terminus a sequence that binds protein of the COPI complex. This suggests that these proteins may be trafficking between the ER and the Golgi apparatus.

The fourth group contains NSTs that transport UDP-gal and UDP-glcNAc, but also transporters for CMP-sia. In most of them it is possible to find the highly conserved GL sequence in the sixth putative transmembrane domain (Figure 5). Because mutations in the conserved glycine lead to the inactivation of the transporter, it has been hypothesized that this glycine may play a role in the translocation of the nucleotide sugar rather than in substrate recognition, since it is located both in UDP-gal and in CMP-sia transporters.

The identification of domains which are linked to a transport function should help in the identification of a larger number of NSTs present in the genome of different organisms.

\section{Phenotypic changes associated with nucleotide-sugar transporter defects}

Due to their property of supplying glycosyltransferases with the substrates they need, modifying NST activity may have significant consequences for glycoconjugate structure. NST activity can thus be a rate-limiting step in the glycosylation process. For example, in a mutant cell line deficient in UDP-gal transport (MDCK-RCA), the galactosylation of sphingolipids, proteins and keratan sulfate is reduced (43). In yeast, mutation of a GDP-mannose transporter $(\mathrm{ScVrg} 4 \mathrm{p})$ reduces the mannosylation of cell wall polysaccharides, sphingolipids and glycoproteins (44). Similarly, mutation of the UDP-glcA/gal/galNAc transporter ( $S Q V-7)$ in $C$. elegans results in distinct phenotypic changes in the reproductive organs early in development, affecting fertility and viability
UDP-Gal UDP-Gal/GalNAC

SLC

UDP-Gal

SFR3

CMP-Sia

hUGT1

GMS1

\section{TMD 6}

ASQGFFFGYTPAVWGVVLNQAFGG----ILVAVVVKYADNILKGFATSLSIVLSTVAS-I ATRGFFFGYTPAVWGVVLNQAFGG----LLVAVVVKYADNILKGFATSLSIVLSTVAS-I SKNGFFQGYNRLTWIVVVLQALGG----LVIAAVIKYADNILKGFATSLSIILSTLISYF SKNGFFQGYNRLTWIVVILQALGG----LVIAAVIKYADNILKGFATSLSIILSTLISYF NEYGLLYGFDSIVWLTVLWYGVGG----LSVAVCIKYADNIAKNFATSVAIILSTIGS-I KEKGFFYGYTYYVWEVIFLASVGE----LYTSVVVKYTDNIMKGFSAAAAIVLSTIAS-V SGPGLLEGFSGWAALVVLSQALNG----LLMSAVMKHGSSITRLFVVSCSLVVNAVLS-A QQPLSFINRHPSILKDMLLFACTGSVGQLFIFFTLEKFGSITLVTITLTRKIFTMLLS-V

Figure 5. Highly conserved glycine and leucine are located in a putative transmembrane domain. Alignment using Clustal W. Putative transmembrane domains 6 (TMD6) and 7 (TMD7) are highlighted. The highly conserved residues $G$ and L are boxed in black and other conserved residues are boxed in dark gray. 
(28). In Drosophila, FRC, a multi-specific UDP-sugar transporter, supplies the substrate for the glycosylation of Notch, so that frc mutants have Notch-like phenotypes. In the embryonic stage, ectodermal cells are replaced by neural cells leading to death (like Notch), whereas if expression is conditionally reduced later in development, adults have smaller eyes, and wings with thickened veins and nicked margins (30).

\section{Nucleotide-sugar transporters and human disease}

NSTs are an attractive target for controlling disease caused by microorganisms pathogenic to humans, particularly if they transport substrates not transported by humans. For example, $C$. glabrata is the second most frequently isolated human fungal pathogen after $C$. albicans, causative agents of candidiasis. Both species possess cell wall-associated mannosylated proteins, essential for many biological functions, including pathogenic attachment and interaction with host cells (45). These mannoproteins are produced in the lumen of the Golgi apparatus, and both species code for an NST related to $\mathrm{ScVrg} 4 \mathrm{p}$ of $S$. cerevisiae which is capable of transporting GDP-man into the lumen of this compartment $(37,38)$. Mutations in $\mathrm{CaVrg} 4$ or $\mathrm{CgVrg}_{4}$ are lethal, highlighting not only the importance of the mannoproteins for cell survival, but also that there is just a single functional GDP-man transporter in these microorganisms. Since GDP-man transport is not required for glycoconjugate modification in the human Golgi apparatus, drugs specifically targeted at depleting the activity of $\mathrm{CaVrg} 4 \mathrm{p}$ or $\mathrm{CgVrg} 4 \mathrm{p}$ could be developed for potential therapeutic use. However, such a chemotherapeutic strategy may not be effective in combating all types of infection. For example, L. donovani, the causative agent of the most severe form of leishmaniasis, possesses a multi-specific GDP-sugar transporter, Lpg2p, essential for the synthesis of the cell surface glycolipid lipophosphoglycan $(4,26,46)$. However, C3PO L. donovani mutants, which lack LPG2p, are viable, indicating that, unlike ScVrg4, CaVrg4, and $C g V r g 4, L P G 2$ is not an essential gene (4). Interestingly, mutation in the corresponding gene of L. major significantly reduced the survival of the parasite in its natural sand-fly (Phlebotomus papatasi) vector host (47), indicating a possible method of control.

Furthermore, the activity of endogenous NSTs has been implicated in the development of a variety of human diseases. For example, there were more abundant transcripts specifically of a UDP-gal NST, but not of transporters for UDP-glcNAc or CMPsia obtained from colonic cancer cells compared to non-malignant tissue obtained from the same patients (48). Increased levels of UDP-gal transporter activity increased the expression of galactose-containing markers of colonic cancers such as the sialyl Lewis A and $\mathrm{X}$ antigens and the Thomsen-Friedenreich antigen. These carbohydrate determinants are believed to be linked with the metastatic nature of these cells and the cell adhesion qualities of cancerous cells are altered (48). These results suggest that the UDP-gal transporter participates in hematogenous metastasis and would be a good target for chemotherapy of colon cancer after specific inhibitors have been developed.

An increasing number of congenital disorders of glycosylation (CDG) have been recently described (49). Two of these alterations have been shown to be directly associated with a defect in a human NST. Patients with leukocyte adhesion deficiency type II (LADII/CDG-IIc) are characterized by a flat face, and suffer retarded growth and frequent bacterial infection, with persistently elevated peripheral leukocytes. All fucosecontaining cell surface glycoconjugates (except $O$-fucosylated glycans) are absent in LADII patients, including sialyl Lewis $\mathrm{X}$ antigen. This antigen has been implicated in the leukocyte selectin adhesion interaction 
and could account for the observation that the patients are immunocompromised. Biochemical studies have revealed that there was a deficiency in the import of GDPfucose into the Golgi lumen in a patient of Turkish origin (50). Two groups independently showed that the molecular defect was due to a point mutation (R147C) in a GDPfucose transporter $(51,52)$. When fibroblasts from the CDG-IIc patient were transfected with the wild-type copy of the transporter, normal fucosylation patterns were restored (51). Interestingly, several patients of ArabIsraeli origin carry a different point mutation (T308R) (52). Analysis of the biochemical properties of the mutated GDP-fuc transporter in these patients revealed that it had a lower $\mathrm{V}_{\max }$, but equal $K_{\mathrm{m}}$ compared to the wild-type transporter (53).

A few years ago, a patient displaying symptoms of a new syndrome, CDG-IIf, was described. The patient possessed a reduced count of enlarged platelets in peripheral blood (macrothrombocytopenia) and suffered recurrent hemorrhaging, ultimately leading to death at 37 months even after a bone marrow transplant (54). The polymorphonuclear neutrophils were completely lacking in the sialyl Lewis $\mathrm{X}$ antigen, suggesting a deficiency in fucosylation or sialylation. However, the fucosyltransferase and sialyltransferase activities studied were normal, suggesting a potential deficiency in NST activity. cDNAs isolated from the patient and from a control subject were used to transfect Lec 2 cells, a cell line that is deficient in a CMP-sia transporter. The wild-type human cDNA alleles were able to complement the asialo phenotype of the Lec 2 cells, but two alleles from the patient suffering CDG-IIf could not. Se- quencing of the cDNAs from the patient revealed the presence of a premature stop codon and/or the deletion of a sequence leading to partial skipping of exon 6 of the CMP-sia transporter gene (55). These results suggest that the blood phenotypes exhibited by the patient were caused by the reduced availability of Golgi luminal CMPsia as a result of a defective CMP-sia transport across the membrane.

\section{Conclusion}

NSTs are antiporters comprising a gene family that plays a fundamental role in the biosynthesis of glycoconjugates and polysaccharides. They are present in all eukaryotes and are responsible for the transport of nucleotide-sugars from the cytosol into the lumen of the Golgi apparatus, even though some NSTs are also present in the endoplasmic reticulum and it is possible that some NSTs may also be located in other organelles. Given the diversity in glycosylation, an alteration in their normal function may lead to changes in the glycosylation of glycoproteins and glycolipids and in polysaccharide structure, causing in different organisms alterations in development, immune response or virulence of a microorganism. A great deal of information regarding molecular aspects of NSTs has been obtained in recent years; however, we still know little about the regulation of their expression, function and subcellular distribution, as well as their role in vivo and their association with glycosyltransferases. The identification and characterization of a larger number of mutants affected in NSTs may help us to better understand these proteins.

\section{References}

1. Hacker U, Nybakken K, Perrimon N. Heparan sulphate proteoglycans: the sweet side of development. Nat Rev Mol Cell Biol 2005; 6: 530-541.

2. Pilling E, Hofte H. Feedback from the wall. Curr Opin Plant Biol
2003; 6: 611-616.

3. Poulain D, Jouault T. Candida albicans cell wall glycans, host receptors and responses: elements for a decisive crosstalk. Curr Opin Microbiol 2004; 7: 342-349. 
4. Descoteaux A, Luo Y, Turco SJ, Beverley SM. A specialized pathway affecting virulence glycoconjugates of Leishmania. Science 1995; 269: 1869-1872.

5. Seifert GJ. Nucleotide sugar interconversions and cell wall biosynthesis: how to bring the inside to the outside. Curr Opin Plant Biol 2004; 7: 277-284.

6. Hirschberg CB, Snider MD. Topography of glycosylation in the rough endoplasmic reticulum and Golgi apparatus. Annu Rev Biochem 1987; 56: 63-87.

7. Kuhn NJ, White A. The topography of lactose synthesis. Biochem J 1975; 148: 77-84.

8. Kuhn NJ, White A. Evidence for specific transport of uridine diphosphate galactose across the Golgi membrane of rat mammary gland. Biochem J 1976; 154: 243-244.

9. Kuhn NJ, White A. The role of nucleoside diphosphatase in a uridine nucleotide cycle associated with lactose synthesis in rat mammarygland Golgi apparatus. Biochem J 1977; 168: 423-433.

10. Waldman BC, Rudnick G. UDP-GlcNAc transport across the Golgi membrane: electroneutral exchange for dianionic UMP. Biochemistry 1990; 29: 44-52.

11. Hirschberg CB. Topography of glycosylation in the rough endoplasmic reticulum and Golgi apparatus. In: Berger EG, Roth J (Editors), The Golgi apparatus. Basel: Birkhäuser Verlag; 1997.

12. Munoz P, Norambuena L, Orellana A. Evidence for a UDP-glucose transporter in Golgi apparatus-derived vesicles from pea and its possible role in polysaccharide biosynthesis. Plant Physiol 1996; 112: 1585-1594.

13. Perez M, Hirschberg CB. Topography of glycosylation reactions in the rough endoplasmic reticulum membrane. J Biol Chem 1986; 261: 6822-6830.

14. Wulff C, Norambuena L, Orellana A. GDP-fucose uptake into the Golgi apparatus during xyloglucan biosynthesis requires the activity of a transporter-like protein other than the UDP-glucose transporter. Plant Physiol 2000; 122: 867-877.

15. Abeijon C, Robbins PW, Hirschberg CB. Molecular cloning of the Golgi apparatus uridine diphosphate-N-acetylglucosamine transporter from Kluyveromyces lactis. Proc Natl Acad Sci U S A 1996; 93: 5963-5968.

16. Eckhardt M, Gerardy-Schahn R. Molecular cloning of the hamster CMP-sialic acid transporter. Eur J Biochem 1997; 248: 187-192.

17. Miura N, Ishida N, Hoshino M, Yamauchi M, Hara T, Ayusawa D, et al. Human UDP-galactose translocator: molecular cloning of a complementary DNA that complements the genetic defect of a mutant cell line deficient in UDP-galactose translocator. J Biochem 1996; 120: 236-241.

18. Gerardy-Schahn R, Oelmann S, Bakker H. Nucleotide sugar transporters: biological and functional aspects. Biochimie 2001; 83: 775782.

19. Ishida N, Kawakita M. Molecular physiology and pathology of the nucleotide sugar transporter family (SLC35). Pflügers Arch 2004; 447: 768-775.

20. Baldwin TC, Handford MG, Yuseff MI, Orellana A, Dupree P. Identification and characterization of GONST1, a Golgi-localized GDPmannose transporter in Arabidopsis. Plant Cell 2001; 13: 22832295.

21. Norambuena L, Marchant L, Berninsone $P$, Hirschberg CB, Silva $H$, Orellana A. Transport of UDP-galactose in plants. Identification and functional characterization of AtUTr1, an Arabidopsis thaliana UDPgalactos/UDP-glucose transporter. J Biol Chem 2002; 277: 3292332929.

22. Eckhardt M, Gotza B, Gerardy-Schahn R. Membrane topology of the mammalian CMP-sialic acid transporter. J Biol Chem 1999; 274: 8779-8787.

23. Gao XD, Dean N. Distinct protein domains of the yeast Golgi GDPmannose transporter mediate oligomer assembly and export from the endoplasmic reticulum. J Biol Chem 2000; 275: 17718-17727.

24. Berninsone $P$, Eckhardt M, Gerardy-Schahn R, Hirschberg CB. Functional expression of the murine Golgi CMP-sialic acid transporter in Saccharomyces cerevisiae. J Biol Chem 1997; 272: 12616 12619.

25. Norambuena L, Nilo R, Handford M, Reyes F, Marchant L, Meisel L, et al. AtUTr2 is an Arabidopsis thaliana nucleotide sugar transporter located in the Golgi apparatus capable of transporting UDP-galactose. Planta 2005; 222: 521-529.

26. Hong K, Ma D, Beverley SM, Turco SJ. The Leishmania GDPmannose transporter is an autonomous, multi-specific, hexameric complex of LPG2 subunits. Biochemistry 2000; 39: 2013-2022.

27. Segawa H, Soares RP, Kawakita M, Beverley SM, Turco SJ. Reconstitution of GDP-mannose transport activity with purified Leishmania LPG2 protein in liposomes. J Biol Chem 2005; 280: 20282035.

28. Berninsone PM, Hwang HY, Zemtseva I, Horvitz HR, Hirschberg CB. SQV-7, a protein involved in Caenorhabditis elegans epithelial invagination and early embryogenesis, transports UDP-glucuronic acid, UDP-N-acetyl galactosamine, and UDP-galactose. Proc Natl Acad Sci U S A 2001; 98: 3738-3743.

29. Selva EM, Hong K, Baeg GH, Beverley SM, Turco SJ, Perrimon N, et al. Dual role of the fringe connection gene in both heparan sulphate and fringe-dependent signalling events. Nat Cell Biol 2001; 3: 809-815.

30. Goto S, Taniguchi M, Muraoka M, Toyoda H, Sado Y, Kawakita M, et al. UDP-sugar transporter implicated in glycosylation and processing of Notch. Nat Cell Biol 2001; 3: 816-822.

31. Muraoka M, Kawakita M, Ishida N. Molecular characterization of human UDP-glucuronic acid/UDP-N-acetylgalactosamine transporter, a novel nucleotide sugar transporter with dual substrate specificity. FEBS Lett 2001; 495: 87-93.

32. Capasso JM, Hirschberg CB. Effect of nucleotides on translocation of sugar nucleotides and adenosine 3'-phosphate 5'-phosphosulfate into Golgi apparatus vesicles. Biochim Biophys Acta 1984; 777: 133-139.

33. Aoki K, Ishida N, Kawakita M. Substrate recognition by UDP-galactose and CMP-sialic acid transporters. Different sets of transmembrane helices are utilized for the specific recognition of UDP-galactose and CMP-sialic acid. J Biol Chem 2001; 276: 21555-21561.

34. Aoki K, Ishida N, Kawakita M. Substrate recognition by nucleotide sugar transporters: further characterization of substrate recognition regions by analyses of UDP-galactose/CMP-sialic acid transporter chimeras and biochemical analysis of the substrate specificity of parental and chimeric transporters. J Biol Chem 2003; 278: 2288722893.

35. Berninsone PM, Hirschberg CB. Nucleotide sugar transporters of the Golgi apparatus. Curr Opin Struct Biol 2000; 10: 542-547.

36. Gao XD, Nishikawa A, Dean N. Identification of a conserved motif in the yeast Golgi GDP-mannose transporter required for binding to nucleotide sugar. J Biol Chem 2001; 276: 4424-4432.

37. Nishikawa A, Mendez B, Jigami Y, Dean N. Identification of a Candida glabrata homologue of the $S$. cerevisiae VRG4 gene, encoding the Golgi GDP-mannose transporter. Yeast 2002; 19: 691698.

38. Nishikawa A, Poster JB, Jigami Y, Dean N. Molecular and phenotypic analysis of CaVRG4, encoding an essential Golgi apparatus 
GDP-mannose transporter. J Bacteriol 2002; 184: $29-42$.

39. Handford MG, Sicilia F, Brandizzi F, Chung JH, Dupree P. Arabidopsis thaliana expresses multiple Golgi-localised nucleotide-sugar transporters related to GONST1. Mol Genet Genomics 2004; 272: 397-410.

40. Reyes F, Marchant L, Norambuena L, Nilo R, Silva H, Orellana A. AtUTr1, a UDP-glucose/UDP-galactose transporter from Arabidopsis thaliana, is located in the endoplasmic reticulum and up-regulated by the unfolded protein response. J Biol Chem 2006; 281: 9145-9151.

41. Schroder M, Kaufman RJ. The mammalian unfolded protein response. Annu Rev Biochem 2005; 74: 739-789.

42. Parodi AJ. Protein glucosylation and its role in protein folding. Annu Rev Biochem 2000; 69: 69-93.

43. Toma L, Pinhal MA, Dietrich CP, Nader HB, Hirschberg CB. Transport of UDP-galactose into the Golgi lumen regulates the biosynthesis of proteoglycans. J Biol Chem 1996; 271: 3897-3901.

44. Poster JB, Dean N. The yeast VRG4 gene is required for normal Golgi functions and defines a new family of related genes. $J$ Biol Chem 1996; 271: 3837-3845.

45. Chaffin WL, Lopez-Ribot JL, Casanova M, Gozalbo D, Martinez JP. Cell wall and secreted proteins of Candida albicans: identification, function, and expression. Microbiol Mol Biol Rev 1998; 62: 130-180.

46. Ma D, Russell DG, Beverley SM, Turco SJ. Golgi GDP-mannose uptake requires Leishmania LPG2. A member of a eukaryotic family of putative nucleotide-sugar transporters. J Biol Chem 1997; 272: 3799-3805.

47. Spath GF, Lye LF, Segawa H, Sacks DL, Turco SJ, Beverley SM. Persistence without pathology in phosphoglycan-deficient Leishmania major. Science 2003; 301: 1241-1243.

48. Kumamoto K, Goto Y, Sekikawa K, Takenoshita S, Ishida N,
Kawakita M, et al. Increased expression of UDP-galactose transporter messenger RNA in human colon cancer tissues and its implication in synthesis of Thomsen-Friedenreich antigen and sialyl Lewis A/X determinants. Cancer Res 2001; 61: 4620-4627.

49. Jaeken J, Carchon H. Congenital disorders of glycosylation: a booming chapter of pediatrics. Curr Opin Pediatr 2004; 16: 434-439.

50. Lubke T, Marquardt T, von Figura K, Korner C. A new type of carbohydrate-deficient glycoprotein syndrome due to a decreased import of GDP-fucose into the Golgi. J Biol Chem 1999; 274: 2598625989.

51. Luhn K, Wild MK, Eckhardt M, Gerardy-Schahn R, Vestweber D. The gene defective in leukocyte adhesion deficiency II encodes a putative GDP-fucose transporter. Nat Genet 2001; 28: 69-72.

52. Lubke T, Marquardt T, Etzioni A, Hartmann E, von Figura K, Korner C. Complementation cloning identifies CDG-Ilc, a new type of congenital disorders of glycosylation, as a GDP-fucose transporter deficiency. Nat Genet 2001; 28: 73-76.

53. Sturla L, Puglielli L, Tonetti M, Berninsone P, Hirschberg CB, De Flora A, et al. Impairment of the Golgi GDP-L-fucose transport and unresponsiveness to fucose replacement therapy in LAD II patients. Pediatr Res 2001; 49: 537-542.

54. Willig TN, Breton-Gorius J, Elbim C, Mignotte V, Kaplan C, Mollicone $\mathrm{R}$, et al. Macrothrombocytopenia with abnormal demarcation membranes in megakaryocytes and neutropenia with a complete lack of sialyl-Lewis-X antigen in leukocytes - a new syndrome? Blood 2001; 97: 826-828.

55. Martinez-Duncker I, Dupre T, Piller V, Piller F, Candelier JJ, Trichet $C$, et al. Genetic complementation reveals a novel human congenital disorder of glycosylation of type II, due to inactivation of the Golgi CMP-sialic acid transporter. Blood 2005; 105: 2671-2676. 